\title{
Wave-Vector-Dependent Tunneling through Magnetic Barriers
}

\author{
A. Matulis* and F.M. Peeters ${ }^{\dagger}$ \\ Departement Natuurkunde, Universiteit Antwerpen (UIA), Universiteitsplein 1, B-2610 Antwerpen, Belgium \\ P. Vasilopoulos ${ }^{\ddagger}$ \\ Department of Physics, Concordia University, Montréal, Québec, Canada H3G 1M8
}

(Received 24 September 1993)

\begin{abstract}
New tunneling structures are proposed consisting of magnetic barriers that can be created by lithographic patterning of ferromagnetic or superconducting films. The form of the equivalent potential for such a barrier depends on the wave vector of the incident electron. This renders the transmission through such structures an inherently 2D process since the tunneling probability depends not only on the electron's momentum perpendicular to the tunneling barrier but also on its momentum parallel to the barrier. Pronounced resonances are obtained for the tunneling probability and the conductance of a resonant tunneling device consisting of such magnetic barriers.
\end{abstract}

PACS numbers: $73.40 . \mathrm{Gk}, 03.65 . \mathrm{Ge}$

The electron motion has been studied extensively in both macroscopically homogeneous and microscopically inhomogeneous (on the $\AA$ scale) magnetic fields. The latter limit pertains to scattering by magnetic impurities. Between these limits lie inhomogeneous magnetic fields on the $\mathrm{nm}$ scale. They have recently been realized with the creation of magnetic dots [1], the patterning of ferromagnetic materials integrated with semiconductors $[2,3]$, and the deposition of type II superconducting materials on conventional heterostructures [4]. In the latter case the magnetic flux lines that penetrate the two-dimensional electron gas (2DEG) act like scattering centers and offer the possibility of studying weak localization and the dynamics of vortices [5]. Also, an out-ofplane magnetization has been realized by the deposition of ultrathin layers of $\mathrm{Fe}$ on $\mathrm{Ag}$ [6].

Theoretically, in addition to Ref. [5] the creation of superlattices by an inhomogeneous magnetic field $(B)$ has been studied [7] as well as transport properties of a weakly and periodically modulated 2DEG [8] with its elementary excitations [9], and electron motion in a stripe of a 2DEG subject to a constant [10] perpendicular $B$ and one in which $B$ increases linearly in one direction [11]. Moreover, various geometric factors that control the magnitude of the demagnetizing field of ferromagnetic materials have been studied [12].

Motivated by this wealth of experimental and theoretical results we consider in this Letter electron tunneling through structures of magnetic barriers and in particular resonant tunneling. In contrast with tunneling through electric barriers, the tunneling probability depends not only on the electron's momentum perpendicular to the tunneling barrier but also on its momentum parallel to the barrier. This renders the tunneling an inherently two-dimensional process and the magnetic barriers possess wave vector filtering properties.

As shown in Fig. 1 a magnetic barrier can be created by the deposition, on top of a heterostructure, of a ferromagnetic stripe with magnetization (a) perpendicular and (b) parallel to the 2DEG, (c) of a conducting stripe with a current driven through it, and (d) of a type I superconducting plate interrupted by a stripe. In all cases the 2DEG is situated at a distance $z_{0}$ below the stripe whose thickness and height are $d$ and $h$, respectively. Integrating Maxwell's equations gives the magnetic field perpendicular to the $2 \mathrm{D}$ system $\left[\mathbf{B}=B(x, z) \mathbf{e}_{z}\right]$ as

$$
B(x, z)=B_{0}\{K(x+d / 2, z)-K(x-d / 2, z)\},
$$

where we have for these four cases: (a) $B_{0}=M_{0} h / d$, $K(x, z)=2 x d /\left(x^{2}+z^{2}\right)$, (b) $B_{0}=M_{0} h / d, K(x, z)=$ $-z d /\left(x^{2}+z^{2}\right)$, (c) $B_{0}=I / d, K(x, z)=\ln \left[\left(x^{2}+z^{2}\right) / d^{2}\right]$, and (d) $B(x, z)=B_{0} \operatorname{Re}\left[1 / \sqrt{1-(x+i z)^{2}}\right]$, with $M_{0}$ the magnetization of the film and $I$ the current driven through the stripe. In all cases it has been assumed that $h / d \ll 1$ and $h / z_{0} \ll 1$. In case (d) $B_{0}$ depends on how much magnetic flux is concentrated in the stripe which can never exceed the critical magnetic field for the superconductor. The magnetic field produced by the stripes, in units of $B_{0}$, is shown in Fig. 2 for three different depths: $z_{0}=0.1$ (solid curve), $z_{0}=0.3$ (dashed curve), and $z_{0}=0.5$ (dotted curve). The smaller $z_{0}$, i.e., the closer the 2DEG is to the stripes, the sharper the magnetic barrier structure. With increasing $z_{0}$ the magnetic field profile becomes gradually smoother. Concurrently with

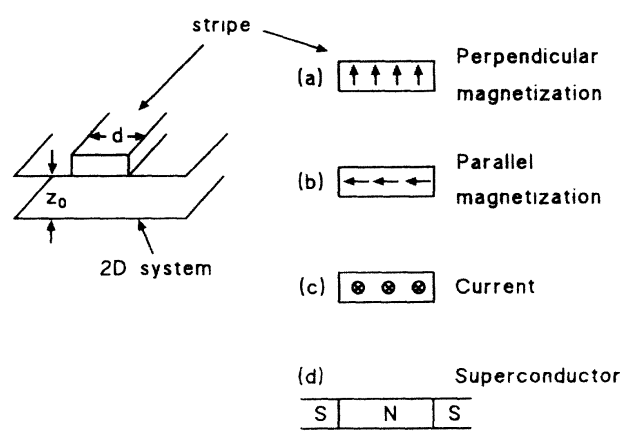

FIG. 1. Layout of the system. 

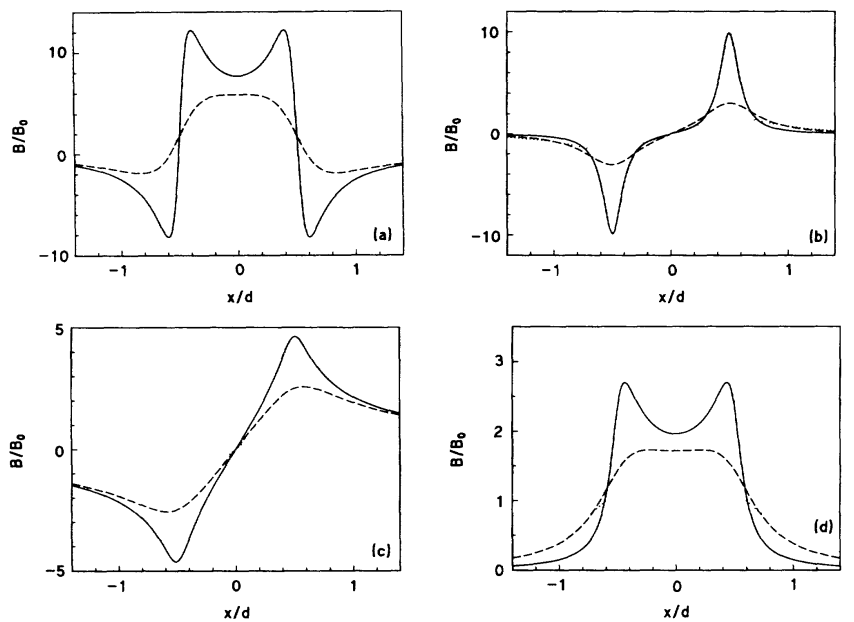

FIG. 2. Magnetic field under the stripe corresponding to the four different configurations as given in Fig. 1. The magnetic field is given at the following distances from the magnetic stripe: $z_{0}=0.1$ (solid curve), $z_{0}=0.3$ (dashed curve), and $z_{0}=0.5$ (dotted curve).

the magnetic field profile one expects a scalar (electric) potential, which can be short circuited by putting a nonmagnetic metallic film [3] between the 2DEG and the patterned layer.

For simplicity we now consider electron tunneling through a magnetic barrier of constant height $B_{0}$ and width $d=x_{+}-x_{-}$surrounded by regions of zero magnetic field. The curves of Fig. 2 show that such a profile can be easily realized. A 2DEG in the $(x, y)$ plane with a magnetic field $\mathbf{B}$ along the $z$ direction is described by the Hamiltonian $H=[\mathbf{p}+e \mathbf{A}(x)]^{2} / 2 m^{*}$ where the vector potential will be taken in the Landau gauge $\mathbf{A}(x)=\left(0, B_{0} x, 0\right)$ which results in $B_{z}=B(x)=$ $d A(x) / d x$. It is convenient to express the results in dimensionless units. For that we use the cyclotron frequency $\omega_{c}=e B_{0} / m^{*}$, where $m^{*}$ is the effective mass of the electron, and $\ell_{B}=\sqrt{\hbar / e B_{0}}$ the magnetic length. We express all quantities in dimensionless units: (1) the magnetic field $B(x) \rightarrow B_{0} B(x)$, (2) the vector potential $A(x) \rightarrow B_{0} \ell_{B} A(x)$, (3) the time $t \rightarrow t \omega_{c}$, (4) the coordinate $\mathbf{r} \rightarrow \ell_{B} \mathbf{r}$, (5) the velocity $\mathbf{v} \rightarrow \ell_{B} \omega_{c} \mathbf{v}$, and (6) the energy $E \rightarrow \hbar \omega_{c} E$. For GaAs and an estimated $B_{0}=0.1$ $\mathrm{T}$ we have $\ell_{B}=813 \AA, \hbar \omega_{c}=0.17 \mathrm{meV}$, and $\ell_{B} \omega_{c}=1.4$ $\mathrm{m} / \mathrm{sec}$.

In these dimensionless units the two-dimensional (2D) Schrödinger equation describing an electron with energy $E$ admits solutions of the form $\Psi(x, y)=e^{i q y} \psi(x)$, where $q$ is the wave vector of the electron in the $y$ direction. The wave function $\psi(x)$ satisfies the one-dimensional (1D) Schrödinger equation

$$
\left\{\frac{d^{2}}{d x^{2}}-[A(x)+q]^{2}+2 E\right\} \psi(x)=0,
$$

and the function $V(x)=[A(x)+q]^{2} / 2$ can be interpreted as a $q$-dependent electric potential and the magnetic field is given by $B(x)=[d V(x) / d x] / \sqrt{2 V(x)}$.

The free electron wave function on the left side of the barrier $\left(x<x_{-}\right)$is $\psi_{-}(x)=A e^{i k_{-}\left(x-x_{-}\right)}+B e^{-k_{-}\left(x-x_{-}\right)}$ and on the right side of it $\left(x>x_{+}\right) \psi_{+}(x)=e^{i k_{+}\left(x-x_{+}\right)}$, where $k_{ \pm}=\sqrt{2[E-V( \pm \infty)]}$ is the $x$ component of the electron wave vector on the corresponding side of the barrier. Under the barrier there are two solutions for $\psi(x)$ which can be written as a linear combination of the Weber function $D_{p}(x)$ and its derivative $D_{p}^{\prime}(x)$. Next we construct the transition matrix

$$
T\left(x, x_{0}\right)=\left(\begin{array}{cc}
u(x) & v(x) \\
u^{\prime}(x) & v^{\prime}(x)
\end{array}\right),
$$

where we defined the functions $u(x)=c\left\{D_{p}^{\prime}(\sqrt{2 q}) D_{p}(z)\right.$ $\left.+D_{p}^{\prime}(-\sqrt{2 q}) D_{p}(-z)\right\}$ and $v(x)=c\left\{D_{p}(\sqrt{2 q}) D_{p}(z)-\right.$ $\left.D_{p}(-\sqrt{2 q}) D_{p}(-z)\right\}$, with $p=E-1 / 2$ and $z=\sqrt{2}(x-$ $q$ ), which satisfies the boundary conditions $u\left(x_{0}\right)=$ $1, u^{\prime}\left(x_{0}\right)=0, v\left(x_{0}\right)=0$, and $v^{\prime}\left(x_{0}\right)=1$. Matching the wave function at the edges of the barrier, $x_{ \pm}$, by means of the above matrix we obtain

$$
A=T_{11}^{-1}+\frac{k_{+}}{k_{-}} T_{22}^{-1}+i\left(\frac{1}{k_{-}} T_{21}^{-1}-k_{+} T_{12}^{-1}\right) ;
$$

the electron transmission through the barrier $t(E, q)$ is
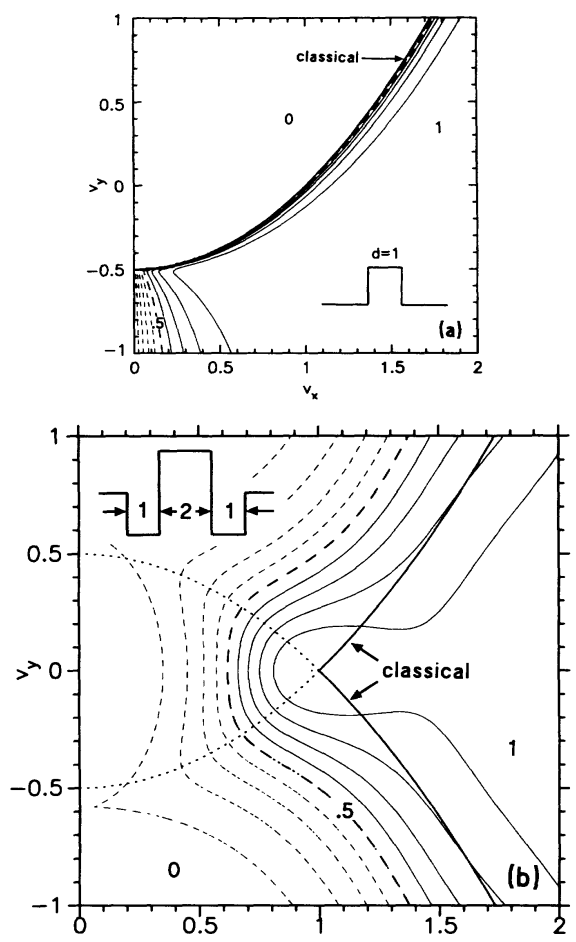

FIG. 3. Contour plot of the electron transmission probability in the $\left(v_{x}, v_{y}\right)$ plane for (a) a simple rectangular magnetic barrier, and (b) a more complex structure. The magnetic field profiles of the corresponding magnetic barriers are shown in the insets. 

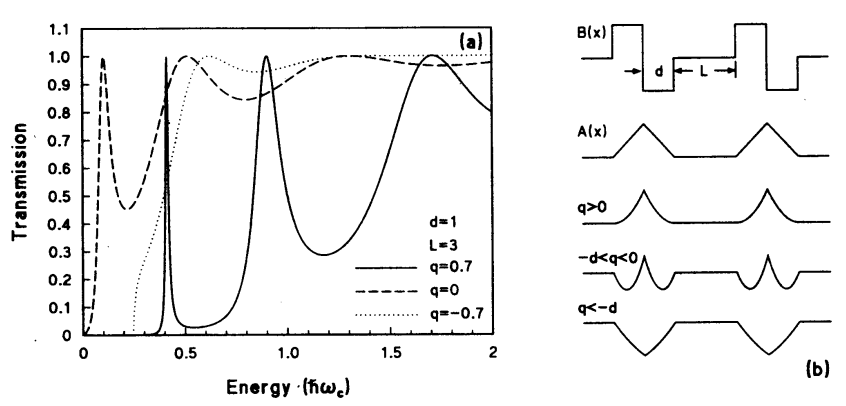

FIG. 4. The transmission probability (a) through the structure given in (b) for different values of the $y$ component of the electron wave vector $(q)$. In (b) we depict the magnetic field $B(x)$, the vector potential $A(x)$, and the corresponding potential $V(x)$ for different $q$ values.

given by

$$
t(E, q)=k_{+} / k_{-}|A|^{2},
$$

where $T^{-1}$ stands for the inverse of the matrix $T \equiv$ $T\left(x_{+}, x_{-}\right)$. For complex structures involving several barriers of constant height, the total $T$ matrix is a product of the $T$ matrices that correspond to the separate barriers and the one describing the free electron propagation between the barriers. As for the electron current through such a structure, it can be calculated, in the ballistic regime, by introducing the conductance $G$ as the electron flow averaged over half the Fermi surface [13]

$$
G=G_{0} \int_{-\pi / 2}^{\pi / 2} t\left(E_{F}, \sqrt{2 E_{F}} \sin \phi\right) \cos \phi d \phi
$$

where $\phi$ is the angle of incidence relative to the $x$ direction. Further, $G_{0}=e^{2} m v_{F} \ell / \hbar^{2}$, where $\ell$ is the length of the structure in the $y$ direction and $v_{F}$ the Fermi velocity.

To reveal the main qualitative features of tunneling through these barriers we restrict our consideration to (i) a single barrier, as shown in the inset of Fig. 3(a), and (ii) complex structures composed of rectangular magnetic barriers as shown in the inset of Fig. 3(b) and in Fig. 4.

(i) Single barrier.-In this case the magnetic tunneling problem is analogous to that of electron transmission above a potential well [14]. The transmission, as a function of the electron energy, shows some weak oscillations. Only for $q=0$, when the potential $V(x)$ is symmetric, and for specific values of the barrier width, does the transmission have some resonant structure in the low energy region due to the presence of a virtual level above the quantum well.

An important feature of the present problem is the transmission anisotropy clearly seen in Fig. 3(a) where we show a contour plot of the transmission in the electron velocity component plane $\left(v_{x}=k_{-}, v_{y}=q+\right.$ $A(-\infty))$. As can be seen the single barrier possesses well-pronounced wave vector filtering properties which can be achieved using quantum point contacts. It is
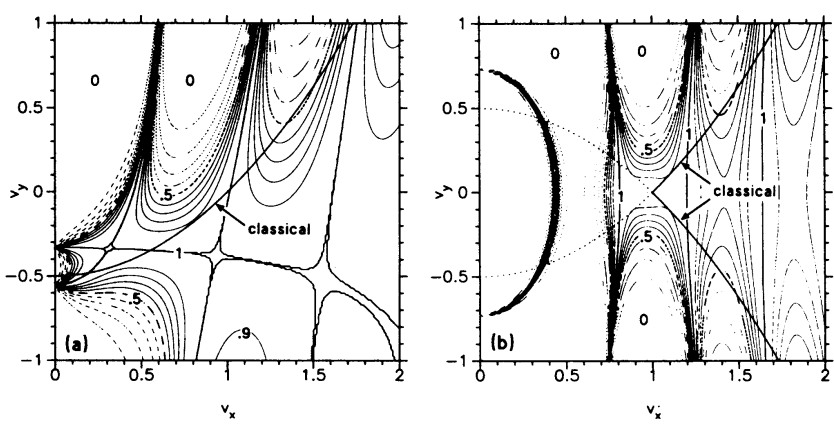

FIG. 5. Contour plot of the electron transmission probability in the $\left(v_{x}, v_{y}\right)$ plane for (a) the structure of Fig. 4 , and (b) the resonant tunneling structure composed of the complex barrier structure of Fig. 3(b) in which the barriers are separated by the distance $L=3$.

interesting to compare this quantum tunneling contour plot with the classical one, represented by the thick solid curve in Fig. 3(a), that is obtained from energy and momentum conservation laws. It is seen that the transmission probability is zero above the thick solid curve $v_{y}=f\left(v_{x}\right)=\left(v_{x}^{2}-d^{2}\right) / 2 d$ and 1 below it. The two results differ slightly only in a narrow region. This is not the case for more complex magnetic barrier structures.

(ii) Complex structures.-Figure 3(b) shows the contour plot of the transmission through a complex structure (see the inset). In contrast to Fig. 3(a), here the classical and quantum mechanical results differ drastically. This is because in the single barrier the nonclassical effects are only due to a virtual state above the well, whereas for $q=0$ the potential corresponding to the structure of Fig. 3(b) is a double barrier which exhibits "quantum mechanical" resonant tunneling properties.

Figure 4(a) shows the transmission through the resonant tunneling structure, shown in Fig. 4(b), as a function of the energy. We see clearly that the curves in Fig. 4(a) follow closely the potential profile for $V(x)$ shown in Fig. 4(b): for $q>0$ we have mostly transmission through double barriers, i.e., resonances, whereas for $q<0$ the transmission is above a double well. The asymmetry of the transmission is shown in the velocity contour plot of Fig. 5(a) together with the classical result. Similar transmission results are obtained for a resonant tunneling structure composed of two units identical to that of Fig. 3(b) with a zero field region, of length $L=3$, between them. The velocity contour plot is shown in Fig. 5 (b) together with the corresponding classical result. We see sharp resonances, the wave vector filtering properties, and the strong dissimilarity between the quantum and classical results. Again the latter is due to the quantum character of tunneling.

Having seen the transmission results, one may wonder to what extent their structure is reflected in measurable quantities which involve some kind of averaging. In Fig. 6 we show the conductance, as given by Eq. (6), for the 

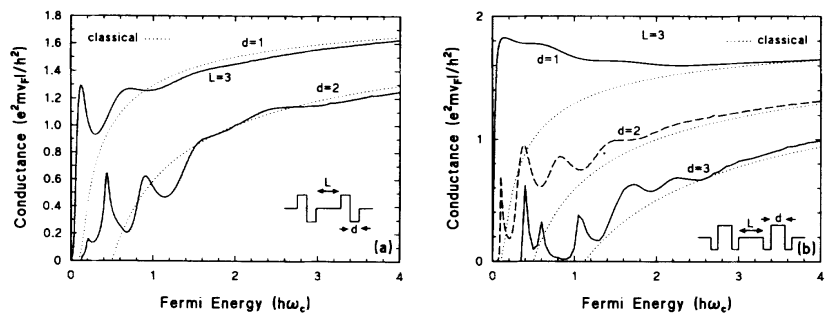

FIG. 6. The conductance through the barrier structure (see inset) of (a) Fig. 3(b), and (b) Fig. 4 for different values of the barrier parameters. The dotted curves show the conductance calculated classically.

structures [15] shown in Fig. 3(b) and Fig. 4 together with the corresponding classical results. Despite the averaging of $t(E, q)$ over half the Fermi surface, we have again strong resonant structure. This structure will become sharper if one can select the wave vectors that give the sharpest resonance in the transmission, cf. Fig. 4. In principle this can be achieved using quantum point contacts. As for the classical results, we see again that they are determined only by the first barrier in each structure.

Although our consideration of electron tunneling through the rectangular magnetic barrier structures gives only a qualitative picture, nevertheless these resonant tunneling spikes should be present in the more realistic cases with barriers of smooth shape, cf. Fig. 2. Indeed these spikes do not depend on the actual shape of the magnetic barrier but only on the presence of barriers in the potential $V(x)$.

In summary, the quantum transmission through magnetic-barrier structures (i) depends not only on the energy but also on the direction of the wave vector, (ii) possesses wave vector filtering properties, (iii) shows wellpronounced resonances whereas the classical one does not, and (iv) is drastically different from the classical transmission which is determined only by the sum of the barriers and is independent of the distance $L$ between them.

This work is supported by the Belgian National Science Foundation (NFWO), the Interuniversity Microelectronics Center (IMEC, Leuven) (F.M.P.), the Collaborative Research Grant NATO-2-205/RG No. 890123 (F.M.P. and P.V.), and the NSERC grant of Canada No. OG-
PIN028 (P.V.). One of us (A.M) acknowledges the support of the EEC through the programme "Cooperation in Science and Technology with Central and Eastern European Countries."

* Permanent address: Semiconductor Physics Institute, Gostauto 11, 2600 Vilnius, Lithuania.

† Electronic address: peeters@nats.uia.ac.be

$\ddagger$ Electronic address: takis@vax2.concordia.ca

[1] M.A. McCord and D.D. Awschalom, Appl. Phys. Lett. 57, 2153 (1990).

[2] M.L. Leadbeater, S.J. Allen, Jr., F. DeRosa, J.P. Harbison, T. Sands, R. Ramesh, L.T. Florez, and V.G. Keramidas, J. Appl. Phys. 69, 4689 (1991); K.M. Krishnan, Appl. Phys. Lett. 61, 2365 (1992); W. Van Roy, E.L. Carpi, M. Van Hove, A. Van Esch, R. Bogaerts, J. De Boeck, and G. Borghs, J. Magn. Magn. Mater. 121, 197 (1993).

[3] R. Yagi and Y. Iye, J. Phys. Soc. Jpn. 62, 1279 (1993).

[4] A.K. Geim, Pis'ma Zh. Eksp. Teor. Fiz. 50, 359 (1989) [JETP Lett. 50, 389 (1990)]; S.J. Bending, K. von Klitzing, and K. Ploog, Phys. Rev. Lett. 65, 1060 (1990).

[5] J. Rammer and A.L. Shelankov, Phys. Rev. B 36, 3135 (1987); A.V. Khaetskii, J. Phys. C 3, 5115 (1991); L. Brey and H.A. Fertig, Phys. Rev. B 47, 15961 (1993).

[6] B.T. Jonker, K.H. Walker, E. Kisher, G.A. Prinz, and C. Carbone, Phys. Rev. Lett. 57, 142 (1986).

[7] D. Yoshioka and Y. Iye, J. Phys. Soc. Jpn. 56, 448 (1987); P.P. Vil'ms and M.V. Éntin, Fiz. Tekh. Poluprovodn. 22, 1905 (1988) [Sov. Phys. Semicond. 22, 1209 (1988)].

[8] P. Vasilopoulos and F.M. Peeters, Superlattices Microstruct. 4, 393 (1990); F.M. Peeters and P. Vasilopoulos, Phys. Rev. B 47, 1466 (1993).

[9] X.-G. Wu and S.E. Ulloa, Phys. Rev. B 47, 7182 (1993).

[10] F.M. Peeters and A. Matulis, Phys. Rev. B 48, 15166 (1993); M. Calvo, Phys. Rev. B 48, 2365 (1993).

[11] J.E. Müller, Phys. Rev. Lett. 68, 385 (1992).

[12] W. Van Roy, J. De Boeck, and G. Borghs, Appl. Phys. Lett. 61, 3056 (1992).

[13] M. Büttiker, Phys. Rev. Lett. 59, 1761 (1986).

[14] A. Matulis, F.M. Peeters, and P. Vasilopoulos, in Proceedings of the International Conference EP2DS-10, Newport, 31 May-4 June 1993 [Surf. Sci. (to be published)].

[15] The conductance for a single barrier shows only a very weak resonance for low energies, see Ref. [14]. 

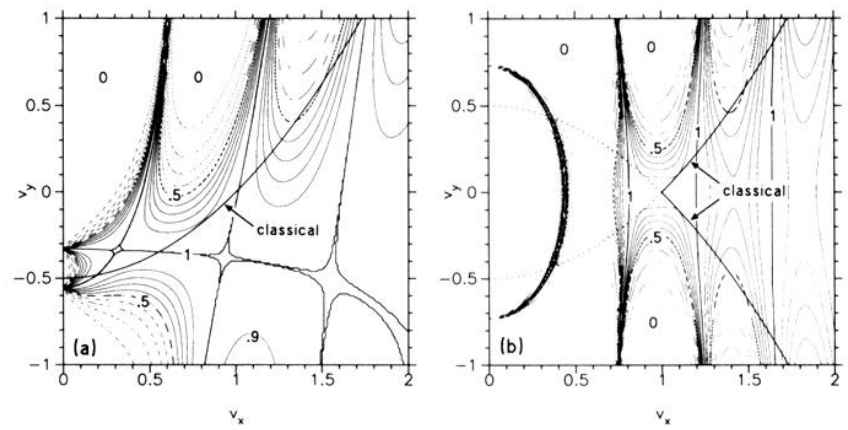

FIG. 5. Contour plot of the electron transmission probability in the $\left(v_{x}, v_{y}\right)$ plane for (a) the structure of Fig. 4, and (b) the resonant tunneling structure composed of the complex barrier structure of Fig. 3(b) in which the barriers are separated by the distance $L=3$. 